Cinémas

Revue d'études cinématographiques

Revue d'études cinématographiques

Journal of Film Studies

\title{
Montage intertextuel et formes contemporaines du remploi dans le cinéma expérimental
}

\section{Nicole Brenez}

Volume 13, numéro 1-2, automne 2002

Limite(s) du montage

URI : https://id.erudit.org/iderudit/007956ar

DOI : https://doi.org/10.7202/007956ar

Aller au sommaire du numéro

Éditeur(s)

Cinémas

ISSN

1181-6945 (imprimé)

1705-6500 (numérique)

Découvrir la revue

Citer cet article

Brenez, N. (2002). Montage intertextuel et formes contemporaines du remploi dans le cinéma expérimental. Cinémas / Revue d'études cinématographiques, 13(1-2), 49-67. https://doi.org/10.7202/007956ar
Résumé de l'article

Essai de cartographie des différentes formes de remploi (montage entre oeuvres) dans le cinéma contemporain. À partir d'une distinction entre remploi intertextuel et recyclage (endogène et exogène), on distingue cinq usages principaux du recyclage : élégiaque ; critique ; structurel ; matériologique ; analytique. Ceux-ci permettent de repérer quelques formes particulièrement travaillées dans le cinéma expérimental, telles que l'anamnèse, le détournement, la glose, la variation, le montage croisé, l'effet double-bande... et, toujours, le ready-made. 


\section{Montage intertextuel et formes contemporaines du remploi dans le cinéma expérimental}

\section{Nicole Brenez}

\section{RÉSUMÉ}

Essai de cartographie des différentes formes de remploi (montage entre œuvres) dans le cinéma contemporain. À partir d'une distinction entre remploi intertextuel et recyclage (endogène et exogène), on distingue cinq usages principaux du recyclage: élégiaque; critique; structurel; matériologique; analytique. Ceux-ci permettent de repérer quelques formes particulièrement travaillées dans le cinéma expérimental, telles que l'anamnèse, le détournement, la glose, la variation, le montage croisé, l'effet double-bande... et, toujours, le ready-made.

\section{ABSTRACT}

A cartographic investigation of the different types of sampling (editing between films) found in contemporary cinema. Drawn from a distinction made between intertextual sampling and recycling (endogenous and exogenous), there are five distinguishable purposes for recycling: elegiac; critical; structural; materialogical; analytical. As a result, it is possible to identify certain forms specifically employed by experimental cinema, such as remembrance, diversion, explanation, variation, plaited montage, the double-track effect... and, indeed, the ready made.

Dans l'histoire de l'art, le remploi constitue probablement la pratique à la fois la plus constante et la plus diverse quant à la 
fabrication des images. Le cinéma n'a cessé d'en intensifier les deux formes:

- le remploi intertextuel, c'est-à-dire in re ("en esprit»), où l'œuvre initiale se voit imitée, en totalité ou par quelque aspect (motif, schème, istoria, etc.). Du Voyage dans la Lune de Segundo de Chomon (1909) reprenant plan par plan celui de Méliès (1902) au Psycho de Gus Van Sant (1999) qui, après quatre décennies de variantes de l'original, retourne à un état supposé littéral du film d'Hitchcock (1960), le cinéma s'est montré capable de formes intertextuelles inventives dont l'histoire reste à établir. On en dégagerait d'ailleurs sans mal les principaux prototypes à partir du corpus hitchcockien: Van Sant travaille comme on enlèverait des couches de vernis et s'efforce d'atteindre en quelque sorte un état neutre du remploi là où, prenant le contre-pied de cette position, Brian DePalma s'interrogeait sur le latent (mode intensif: retrouver l'image cachée, le film devient un examen à l'infrarouge) ou sur le déploiement (mode extensif: développer toutes les images possibles, restituer le spectre des significations, de l'infrarouge à l'ultraviolet).

- le recyclage, ou remploi in se (remploi de la chose même), dont le cinéma a institué certaines formes figées et inauguré quelques autres ${ }^{1}$.

\section{Le recyclage endogène}

Mentionnons que se situent aux deux extrêmes:

- la bande-annonce, pratique industrielle et immédiate du recyclage;

- l'autosynthèse, par laquelle un cinéaste récapitule son œuvre en reprenant des fragments de films antérieurs, souvent pour les inscrire dans un parcours autobiographique, comme dans Film Portrait de Jerome Hill (1970), Dialogue With a Woman Departed de Leo Hurwitz (1972-1980), Un film de Marcel Hanoun (1983) ou Trying to Kiss the Moon de Stephen Dwoskin (1994).

Une initiative singulière pourrait être rapprochée des pratiques en vigueur au début de la généralisation du parlant ${ }^{2}$ :

- la version, dont un exemple capital serait les trois versions du Sicilia! de Jean-Marie Straub et Danièle Huillet (1999), montées à partir des prises différentes d'un même plan. 


\section{Le recyclage exogène}

Sans parler ici des différentes formes de citation ou de convocation, signalons :

- le stock-shot qui, dans le plan, considère essentiellement le motif (pratique répandue dans la série $\mathrm{B}$ américaine où il n'y a pas de seconde équipe pour tourner les plans de situation ou les scènes d'action, ce qui donne lieu à une esthétique, en quelque sorte désinvolte par nécessité, du faux-raccord de texture entre le film d'accueil et le plan interpolé).

- le film de montage, à usage illustratif (mais pas nécessairement mineur) des images déjà tournées, qui s'étend du pamphlet (La Chute de la dynastie des Romanov d'Esfir Choub, 1927, montage critique sur des images d'actualités, source parmi d'autres du cinéma situationniste ou de Notre siècle de Pelechian) jusqu'aux pratiques ordinaires du documentaire télévisé.

- le métadocumentaire, forme typique de la fin du millénaire: un cinéaste trouve ou choisit un morceau de film et s'en sert pour retracer l'ensemble d'une histoire, individuelle, familiale ou collective. Dans le chargeur d'une caméra achetée d'occasion, Henri-François Imbert trouve la bobine non développée d'un film de famille; il part à la recherche de son auteur, des personnages qu'elle représente, et c'est l'occasion de développer une réflexion en images sur le point de vue (Sur la plage de Belfast, 1996). Reprise, où Hervé Le Roux (1996) part à la recherche de l'héroïne révoltée d'un film tourné par Jacques Willemont et Pierre Bonneau en juin 1968, dont le titre pourtant strictement factuel renvoie superbement aux origines (patronales) du cinéma, Reprise du travail aux usines Wonder: c'est l'occasion d'une vaste enquête sur les conditions de vie des travailleurs en usine depuis 1968 jusqu'à nos jours. De telles entreprises, parallèles à celles de Péter Forgács en Hongrie, en disent long sur le rôle des images dans la conscience collective aujourd'hui: plus qu'un corpus d'œuvres, elles constituent un matériau critique pour écrire une histoire dont le cinéma permet désormais d'affirmer qu'on ne l'approfondira jamais assez. Grâce au cinéma, et quitte à perdre l'esprit de synthèse qui prévaut dans l'historiographie littéraire, l'histoire des hommes devient définitivement interminable, au sens où la description authentiquement attentive d'un devenir 
oblige à reconsidérer nos notions d'universalisme, d'histoire collective, d'exemplum et d'individuation.

- le found footage, ou cinéma recyclé, qui possède au moins trois caractéristiques distinctives: il autonomise les images, privilégie l'intervention sur la pellicule considérée comme matériau et s'attache à de nouveaux sites (par exemple, les couches de l'émulsion) ou à de nouvelles formes de montage.

Parmi les sources artistiques des pratiques du found footage, on trouvera notamment le photocollage et le photomontage des années 1920; les formes modernes de l'intertextualité littéraire, de Lautréamont à Joyce et Ezra Pound; le collage cubiste; en musique, «l'éclectisme du brisé", "style de tout le monde après la Seconde Guerre mondiale", comme le formule Adorno (1979, p. 17); et surtout le détournement dadaïste, selon lequel l'art ne consiste plus à produire des reflets et des simulacres, mais à déplacer symboliquement des objets ou des processus. Les notes préparatoires de Fernand Léger (1976, p. 41) pour Le Ballet mécanique représentent sans doute la première esquisse d'un traité du found footage: "[E]mployer des chutes de film quelconque - sans choisir - au hasard.»

Quels sont les principaux usages du found footage? On en proposera cinq, que les œuvres singulières approfondissent, associent ou complètent: élégiaque; critique; structurel ; matériologique; analytique.

\section{A. L’usage élégiaque}

Il s'agit ici de fragmenter un film d'origine, de le démonter, pour n'en conserver que certains moments privilégiés afin de les fétichiser par remontage: le motif prime donc à tel point qu'il subordonne le montage à son apparition, ce qui engendre des formes sauvages de raccordement.

Avec Rose Hobart (1936-1939), Joseph Cornell a livré le chefd'œuvre de l'élégie déconstruite. En ne retenant, par fascination pour l'actrice, que les plans de Rose Hobart dans East of Borneo (George Melford, 1931), il métamorphose un film de genre en portrait onirique de femme et ouvre la voie à une longue descendance. Dans Her Fragant Emulsion (1987) par exemple, portrait de Mimsy Farmer, Lewis Khlar procède également par 
fragmentation, mais aussi par refilmage de la pellicule: il célèbre alors non seulement l'apparence de l'actrice, mais un corps proprement cinématographique. Le portrait ne prétend pas accéder à la femme elle-même; au contraire, les déchirures du montage et les effets d'éloignement dus au refilmage traduisent une mélancolie qui renvoie exclusivement aux principes formels de l'élégie, à commencer par celui du caractère abstrait des figures féminines.

\section{B. L'usage critique}

C'est le plus répandu; il consiste à s'emparer des images de l'industrie cinématographique ou encore des images de sources familiales ou privées pour se livrer à leur détournement voire à leur destruction dans un esprit souvent violent. Dès 1969, Jonas Mekas (1972, p. 350) annonçait la généralisation de ce procédé: "Je pressens que l'ensemble de la production hollywoodienne des quatre-vingts dernières années pourrait devenir un simple matériau pour de futurs artistes cinéastes. »

Plusieurs solutions formelles ont été inventées.

- l'anamnèse: il s'agit de rassembler et d'accoler des images de même nature de façon à leur faire signifier non pas autre chose que ce qu'elles disent, mais exactement ce qu'elles montrent mais que l'on ne veut pas voir. C'est le cas par exemple de Crossing the Great Sagrada (Adrian Brunel, 1924), explicitant sur le mode burlesque l'idéologie colonialiste qui informe les films d'exploration, ou de L'Histoire du soldat inconnu (Henri Storck, 1931), remontant les images d'actualités de 1928-1929 pour dénoncer l'hypocrisie des discours politiques. Plus récemment, Va te faire enculer d'Yves-Marie Mahé (1999), par un simple effet de contamination obtenu avec des films pornographiques, exhume l'obscénité et l'indignité qui règnent dans certaines images de violence universellement admises (Le Parrain, Grease, The Longest Yard, etc.), entreprise que Kirk Tougas n'avait pas totalement menée à bien en confrontant séries télévisées et extermination nazie dans Letter from Vancouver (1973). Davantage que celle d'Arthur Lipsett, l'œuvre de Bruce Conner s'avère exemplaire à cet égard: dans les quelques minutes que durent $A$ Movie (1958), Cosmic Ray (1961) ou Report (1963-1967) passe 
tout l'esprit d'une époque, c'est-à-dire les contradictions ( $A$ Movie) ou les incertitudes (Report) sur lesquelles bute une civilisation.

- le détournement: théorisé et pratiqué par l'Internationale situationniste (nous renvoyons au manifeste de Guy Debord et Gil J. Wolman, "Le détournement", paru en $1956^{3}$ ), on pourrait dire qu'il se simplifie en conversion dans l'œuvre de René Viénet, qui laisse intact le film d'origine et se sert de dialogues burlesques pour lui conférer un sens qu'il n'avait évidemment pas au départ (La dialectique peut-elle casser des briques?, Les Filles de Kamaré, tous deux de 1972).

Il existe aussi des pratiques du détournement sonore, emblématisées en musique par le Come Out de Steve Reich (1966), équivalent acoustique des films de remontage de Rafaël Montañez-Ortiz. La bande-son de Ixe de Lionel Soukaz (1980) représente à cet égard un feu d'artifice sonore, rythmé par les échos ironiques de la "Belle de CadiXXXXXX» de Luis Mariano ou des «Dominique-nique-nique» de sœur Sourire.

- la variation/l'épuisement: ici comme chez Viénet, le travail d'appropriation se concentre sur un seul objet filmique, mais se consacre à le faire varier, voire à en épuiser les potentialités par l'introduction d'un ou de plusieurs éléments plastiques (visuels ou sonores).

Sur la variation sonore, le cas de la Lettre de Sibérie de Chris Marker (1958) reste fameux, mais on peut penser aussi à Lecture d'une image sculptée de Giacometti (J. Poinssac, 1970), qui propose deux montages et deux accompagnements sonores différents pour la description de l'ensemble sculptural Trois hommes qui marchent. Dans l'un et l'autre cas, le travail de variation conclut au caractère inépuisable de l'image d'origine: on pourra toujours lui faire signifier autre chose, elle se révèle par nature polysémique.

Le travail de variation mis en ouvre dans The Politics of Perception de Kirk Tougas (1973), exténuation visuelle et sonore de la bande-annonce d'un film commercial (The Mechanic, Michael Winner, 1973) revenant en boucle une trentaine de fois, est pour sa part purement critique: contre l'empire de la netteté analogique de l'image, The Politics of Perception rappelle la palette très étendue des nuances plastiques possibles et constitue à ce 
titre une toile cinématographique où chaque nuance vient faire événement; contre l'empire de la convention narrative, le film montre, sans doute malgré lui, que la tératologie plastique s'avère plus judicieuse pour rendre justice à un scénario morbide que ne l'était son développement narratif initial; et surtout, contre l'empire des images mortes, le film se livre à une critique de la destruction en passant du noir au blanc: alors que la logique de la défiguration semblait tendre vers l'engloutissement des silhouettes dans le noir, c'est le contraire qui advient, car il ne s'agit plus de détruire des personnages (et donc de s'aligner sur le film d'origine) mais de purifier l'écran, de le laver au moins une fois non seulement de son imagerie, mais du cliché saisi dans sa nature même, c'est-à-dire dans sa puissance de rémanence.

- le ready-made: inverse dans sa technique et pourtant semblable dans les polémiques qu'il suscite, le ready-made déplace entièrement le sens de l'objet sans pourtant lui toucher. Comme chez Duchamp, il existe dans l'histoire du cinéma des readymade purs, tels Perfect Film de Ken Jacobs (1986), Bad Burns de Paul Sharits (assemblage des chutes de son Third Degree), ou Profondeurs mystérieuses: chutes (dans les) de Claudine Kaufmann (1998, utilisation élective d'une copie superbement abîmée du film de Pabst); ainsi que des ready-made arrangés, comme ceux du précurseur Maurice Lemaître (Chutes et Une ouvre, tous deux de 1968), de Dominique Païni (Ruines arrangées, 1984), d'Émeric de Lastens (Terre Adélie, 1999) ou Portrait $n^{\circ} 1$ de Hervé Pichard (2001, retour en boucle ralentie d'un gros plan de visage féminin attaqué de façon différente à chaque photogramme par la décomposition de la copie, comme si le cinéma était pour lui-même son meilleur plasticien). Dans tous ces cas, l'acte créateur consiste à métamorphoser un rebut industriel (rushes, copie endommagée, chutes, amorces, voire film d'actualités comme chez Ken Jacobs) en œuvre d'art, selon le principe d'inversion entre l'accomplissement et le déchet mis en pratique par Isidore Isou dès le Traité de bave et d'éternité (1951). Prisme de Stéfani de Loppinot prend le contre-pied de cette tradition en s'attaquant au Mépris: en prismant la séquence des rushes du film de Godard, il s'agit, à la faveur d'un anagramme duchampien (prisme/mépris), de revivifier plastiquement des 

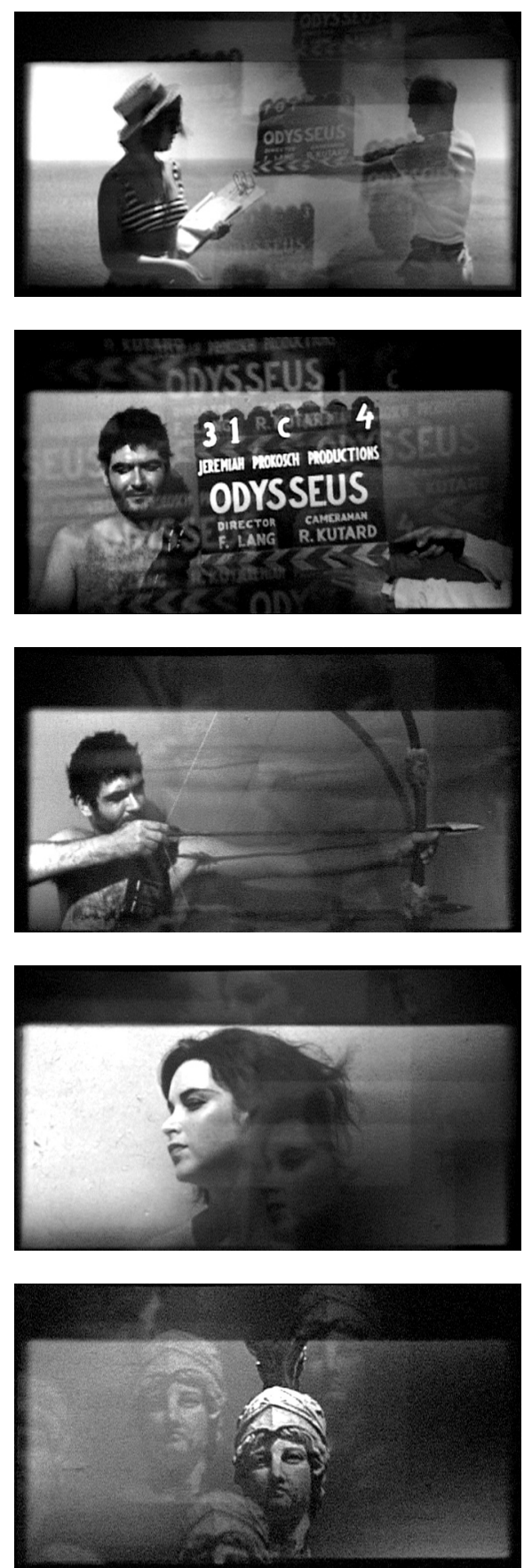

Prisme, Stéfani de Loppinot, 1999

(@ Stéfani de Loppinot) 

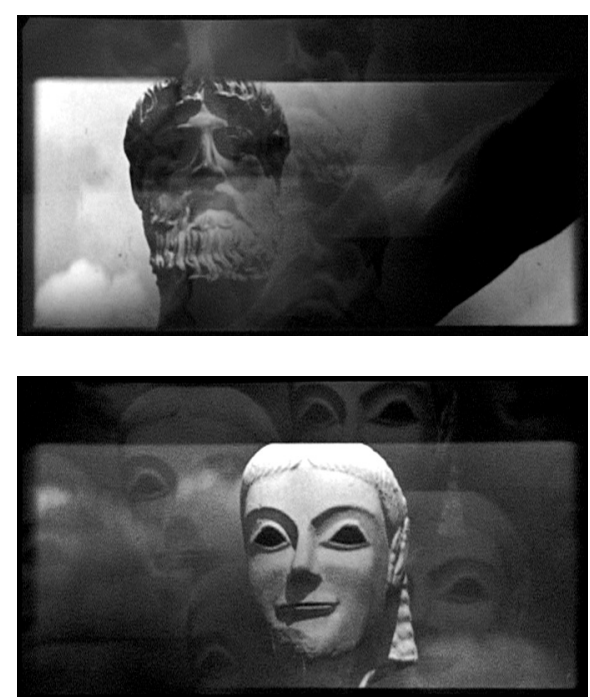

Prisme, Stéfani de Loppinot, 1999

(C) Stéfani de Loppinot)

images désormais guettées par la pétrification académique (y compris à cause du sort que Godard leur réserve dans ses Histoire(s) du cinéma, et en dépit de ce qu'il avait inventé dans Soft and Hard). Grâce à l'usage du prisme, les statues se remettent à bouger, les plans à tournoyer, la beauté se diffracte et recommence à irradier, le monument historique redevient cinétique. Outre son charme visuel, Prisme importe aussi en ce qu'il introduit l'histoire de la réception au registre des éléments que l'on recueille au moment de recycler des images. Une telle prise en charge n'avait auparavant été tentée que par les films de Ken Jacobs, tels The Georgetown Loop et Disoriented Express (1995), dont les basculements et retournements permettaient d'évoquer l'étrangeté éprouvée par les spectateurs contemporains de ces films des premiers temps à la vue de paysages dévorés autant par le chemin de fer que par un cinéma au service de la conquête visuelle du monde.

\section{L'usage structurel}

The Politics of Perception obéit à l'une des règles élémentaires du cinéma structurel: élaborer un film, non pas à partir d'une 
image ou d'un motif, mais d'une proposition, d'un protocole, qui concerne de façon réflexive le cinéma lui-même. L'indifférence (toute relative) au motif s'accommode donc fort bien du found footage, qui en constitue en quelque sorte la preuve concrète.

- les effets secondaires du protocole structurel: enrichissement/ essentialité. En réalité, que ce soit chez Paul Sharits, Standish Lawder, Ernie Gehr ou Malcolm Le Grice, l'exploration des formes du défilement, des variations chromatiques ou des vitesses rétroagit toujours sur le plan mis en boucle, que cette exploration enrichisse voire accomplisse les aspects plastiques du plan (Berlin Horse, 1970, sur le motif du cheval évidemment inaugural pour le cinéma) ou qu'elle retrouve sa nécessité. Dans les chefs-d'œuvre de Peter Gidal, Clouds (1969), ou de Mike Dunford, Silver Surfer (1972), l'exploration du décadrage chez le premier et de la texture de l'image chez le second redonnent un sens à la banalité du motif: de sans cesse frôler le cadre, tantôt hors champ, tantôt in, l'avion de Clouds redevient l'objet fragile menacé par l'illimité que nous ne voulons plus qu'il soit; et le Silver Surfer, englouti dans un grain de pellicule de plus en plus épais, englué dans sa propre disparition, nous restitue les effets d'un voyage magique dans la sensation qui répond bien mieux aux idéaux du surf que n'importe quelle image analogique d'une vague supposée ultime (comme dans Point Break par exemple).

- l'effet double-bande. Les contemporains et les successeurs du cinéma structurel spécialisés dans le found footage ont accentué certains de ces effets-retours du démontage/remontage sur le travail de figuration. Raphaël Montañez-Ortiz puis Martin Arnold, en introduisant de nouvelles partitions dans le défilement des photogrammes, produisent des figures d'automates qui manifestent non seulement de façon réflexive le caractère fondamentalement mécanique sur lequel repose l'illusion analogique, mais sur un registre cette fois figuratif le fonctionnement pulsionnel qui agite les hommes et commande à leurs comportements les plus civilisés (un show d'Elvis Presley chez Montañez-Ortiz ou le baiser donné par l'époux à l'épouse chez Martin Arnold dans Pièce touchée, 1989). 


\section{L'usage matériologique}

Patrice Kirchhofer, Cécile Fontaine, Carl Brown, Jürgen Reble et plus récemment Martha Colburn (I’m Gonna) ou David Matarasso se sont livrés à de grandes explorations des propriétés spécifiques de la pellicule comme matière. Dans ce domaine, quatre grandes possibilités se dégagent naturellement:

- considérer la chimie de l'émulsion (Patrice Kirchhofer, Schmelzdahin, Carl Brown, dont les recherches sur l'altération chimique du celluloïd ont été préfigurées dans un plan visionnaire de Rien que les heures d'Alberto Cavalcanti en 1927);

- décomposer le photogramme en ses couches et souscouches, par un travail de mosaïque dont le Dellamorte Dellamorte Dellamore de David Matarasso (2000) représente un aboutissement plastique qui fait de chaque photogramme une œuvre en soi.

À l'intersection de ces deux tendances, l'œuvre de Cécile Fontaine utilise toutes les ressources possibles de décollage ou de remontage et ne cesse de prouver la nature généreuse de ce matériau qui, avant des artistes comme elle, semblait se réduire à un simple et plat support d'enregistrement.

- Dans Standard Gauge (1984), Morgan Fisher établit un traité du format pelliculaire: ici, le $35 \mathrm{~mm}$, dont le film, au moyen de fragments collectés par l'auteur, nous raconte l'histoire technique, les caractéristiques, la diversité (notamment chromatique) et certaines bizarreries émouvantes, comme des sous-titres mal calés. Toute œuvre de refilmage réinterroge par nature le format des images; dans Standard Gauge, hymne au $35 \mathrm{~mm}$, on touche aussi à une précision historique absolument sans précédent.

- De façon extrêmement subtile, Teresa Faucon se livre à l'examen d'un quatrième site pelliculaire concret, le raccord luimême. Avec Homage Two Tinguely To Tinguely Too (1999), elle inaugure une série dont le motif est l'archétype féminin et le problème celui de la teneur du raccordement cinématographique. Comme l'indique le titre tintinnabulant de ce premier film, à partir d'une séquence de tremblement de terre dans One Million Years B.C., Teresa Faucon opère une répétition fine des changements de plans, qui finissent par faire vibrer non plus 
seulement les motifs (comme dans le film initial) mais aussi les raccords, exacerbés dans leurs propriétés de cassure et de résonance: alors même que les changements de plans restaient parfaitement transparents dans le film d'origine, ils font figure de séisme dans la tranquillité mimétique de l'analogie, ce qui est bien, en effet, l'événement majeur introduit par le cinéma dans l'histoire de la représentation figurative. Le cinéma est entièrement réplique: reflet des choses, réponse au monde, mais aussi au sens sismique d'un tremblement objectif permanent et d'un bouleversement toujours possible des phénomènes.

\section{E. L'usage analytique}

Sur le modèle d'une investigation scientifique, mais capables d'en déborder ou d'en subvertir la rationalité, certains auteurs ont choisi un objet ou un fait filmique et se sont consacrés à l'étudier de façon approfondie. Selon les moyens employés, il existe au moins quatre voies pour cette sorte d'étude visuelle:

- la glose: solution radicale par sa simplicité, elle a été mise en œuvre par Maurice Lemaître dans Erich Von Stroheim (1979). Maurice Lemaître s'est procuré une copie de Foolish Wives et lui a accolé une bande-son où il décrit son admiration pour Stroheim comme auteur et comme acteur. Dans son témoignage inhabituel de respect et d'amour, Maurice Lemaître n'intervient que très progressivement et très peu sur la pellicule, comme si pour une fois, plus que de les détourner et de les transformer, il était révolutionnaire et créateur de montrer les images dans leur clarté propre. Là où l'industrie et le commerce n'ont cessé de réduire et de dégrader l'œuvre de Stroheim, y compris quantitativement (les 42 bobines initiales de Greed auraient fait du film un chef-d'œuvre expérimental), l'hommage de Maurice Lemaitre affirme les valeurs de l'intégralité et de l'intégrité.

- le montage croisé: il s'agit d'éclairer certaines images en recourant à d'autres, que cela s'accompagne ou non d'un commentaire sonore, comme dans toutes les grandes entreprises poétiques d'histoires du cinéma par lui-même: celles d'Al Razutis au Canada (Visual Essays, 1973-1984), de Maurice Lemaître (The Great Train of History, 1978) et de Jean-Luc Godard (Histoire(s) du cinéma, 1978-1998) en France, de Bill Morrison aux États- 
Unis (The Death Train, 1993, The Film of Her, 1996), de Gustav Deutsch en Autriche (Film ist, 1998), etc.

- la variation analytique: grâce à son travail de retour plastique différentiel, Tom Tom the Piper's Son de Ken Jacobs (19691971) envisage le mouvement de trois manières: comme trajet objectif, comme passage et comme circulation psychique; puis il traque le mouvement dans et entre les motifs, dans et entre les plans, dans et entre les vitesses, au sein de chaque intervalle (jusque dans le passage d'un photogramme à l'autre) et d'un intervalle à l'autre. En faisant varier la distance à l'image (prise tantôt comme motif, tantôt comme cadre, tantôt comme photogramme), il en révèle le cinétisme intégral — toutes les sources, tous les sites du mouvement, ainsi que ses différents régimes: défilement (de la pellicule), défilé (des photogrammes), animation (des motifs), agitation (des grains), hachuré (des limites, cadre, barre des photogrammes, montage), happé (des rimes plastiques qui traversent l'ensemble), nappé (de l'événementialité optique qui ne cesse de se présenter à nous).

- la synthèse du montage croisé et de la variation analytique. Si avec Tom Tom Ken Jacobs explicite la morphologie de l'image filmique, Yervant Gianikian et Angela Ricci-Lucchi Gianikian s'attachent à en restituer une autre dimension, complexe et subtile, celle de sa nature historique. En procédant par refilmage (d'images qu'ils ont eux-mêmes restaurées), par ralentissement et souvent par chromatisation, ils libèrent les plans des effets de fugacité inhérents à l'enregistrement documentaire. L'image n'est plus un éclat aléatoire et fragile arraché au cours de l'histoire, elle se charge d'une nécessité qui permet à chaque motif, soldat, animal, groupe, paysage, d'établir le constat de son irremplaçable avoir-été-là et de devenir le sujet de sa propre défection. Comme le formule si bien Bernard Benoliel (2000, p. 94): «Un plan documentaire refilmé par les Gianikian ressemble à une "vue" Lumière mais dont l'opérateur serait Étienne-Jules Marey." Les films des Gianikian, pamphlets politiques contre toutes les formes d'exploitation (colonialiste, militaire, scientifique, etc.) en même temps que déplorations auratiques, inventent une forme d'épiphanie critique.

Une synthèse monumentale de l'ensemble des procédés, des usages et des effets a été effectuée par Ken Jacobs avec Tom Tom 

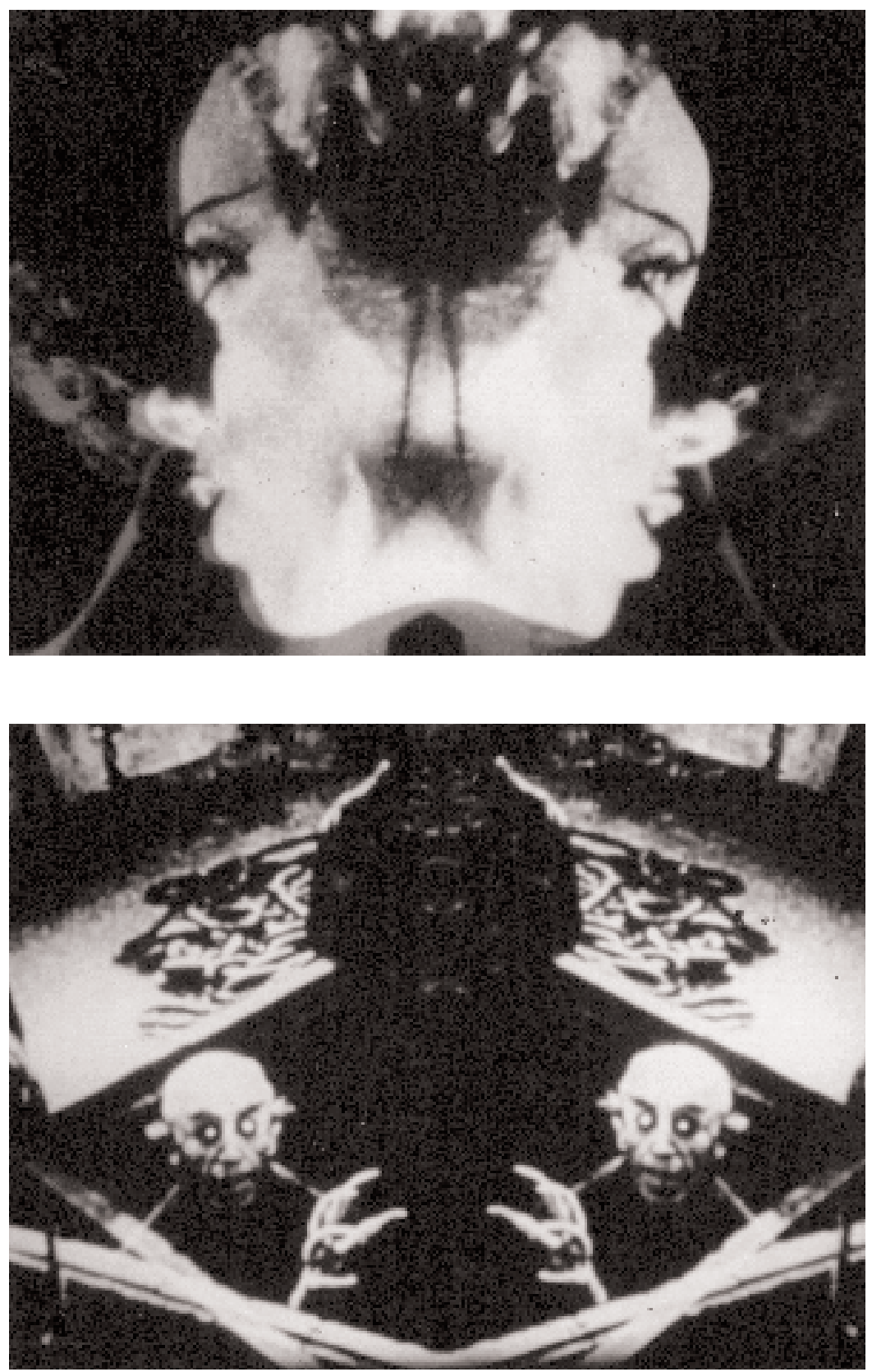

Visual Essays: Origins of Film, Al Razutis, 1973-1984 (C) Al Razutis). D'autres images du film sont disponibles à www.alchemists.com/visual_ alchemy/film_cat.htm. 

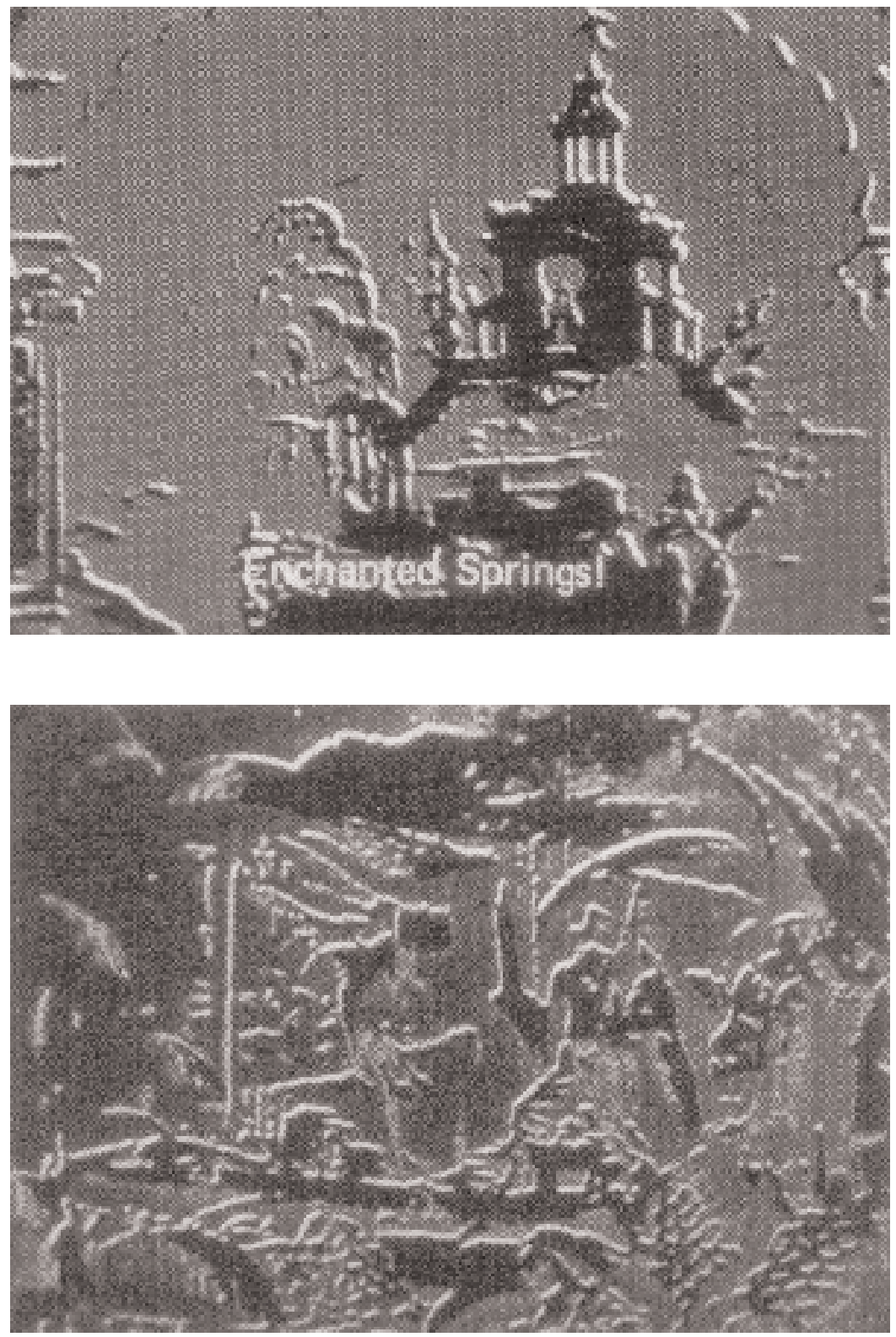

Visual Essays: Origins of Film, Al Razutis, 1973-1984 (ㄷ Al Razutis). D'autres images du film sont disponibles à www.alchemists.com/visual_ alchemy/film_cat.htm. 

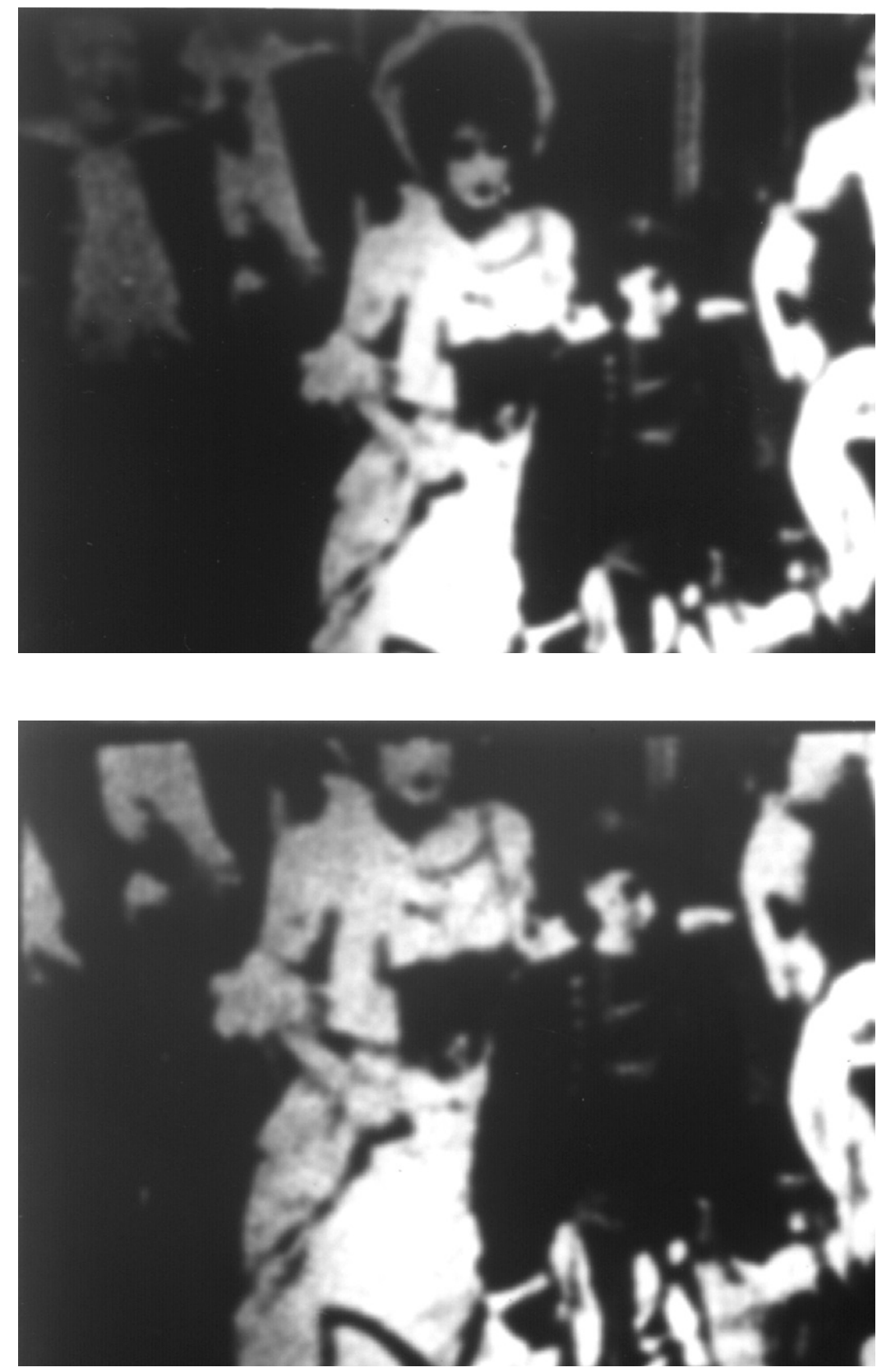

Tom Tom the Piper's Son, Ken Jacobs, 1969-1971

64 CiNéMAS, vol. 13, nºs 1-2 

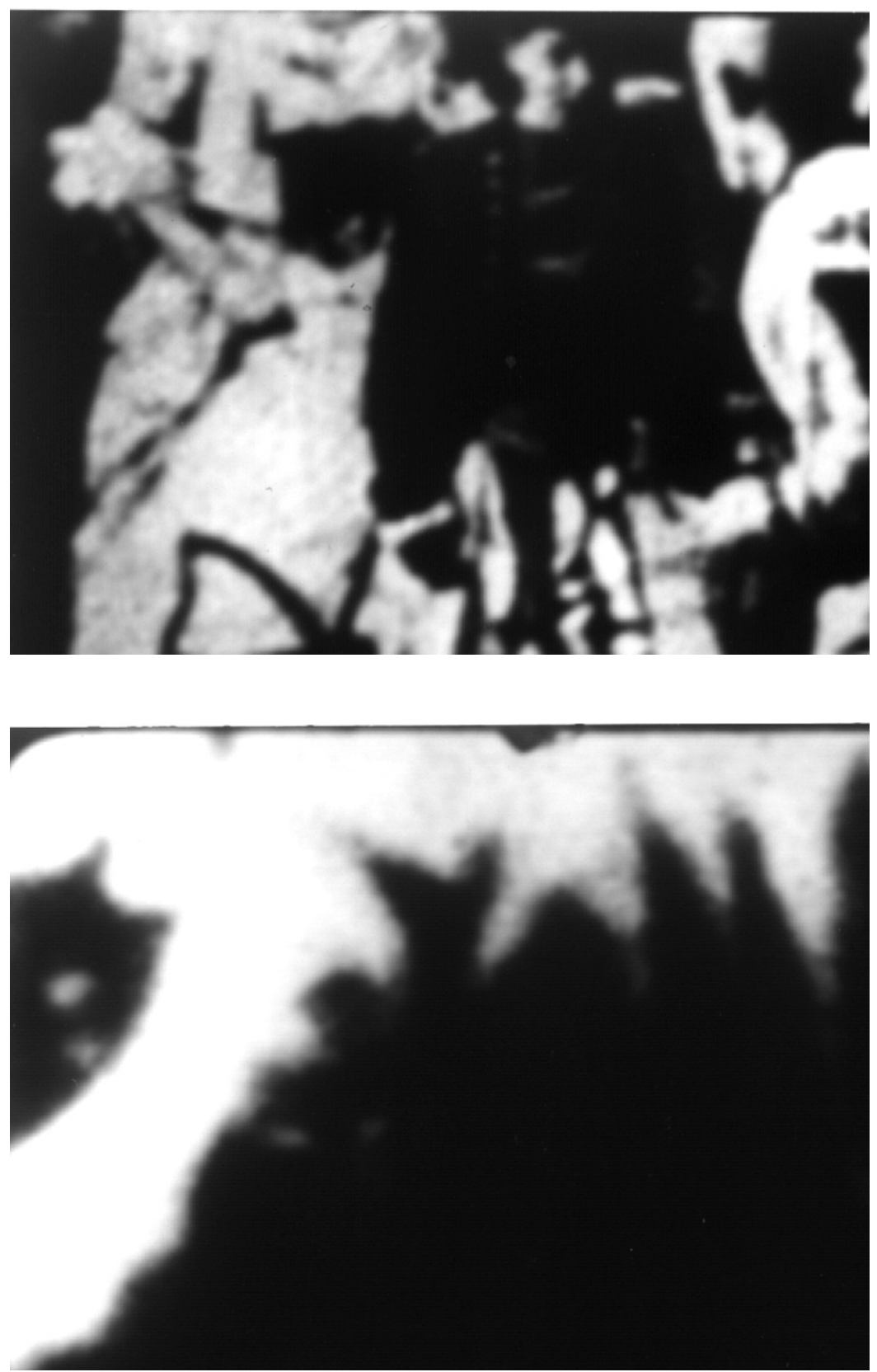

Tom Tom the Piper's Son, Ken Jacobs, 1969-1971 


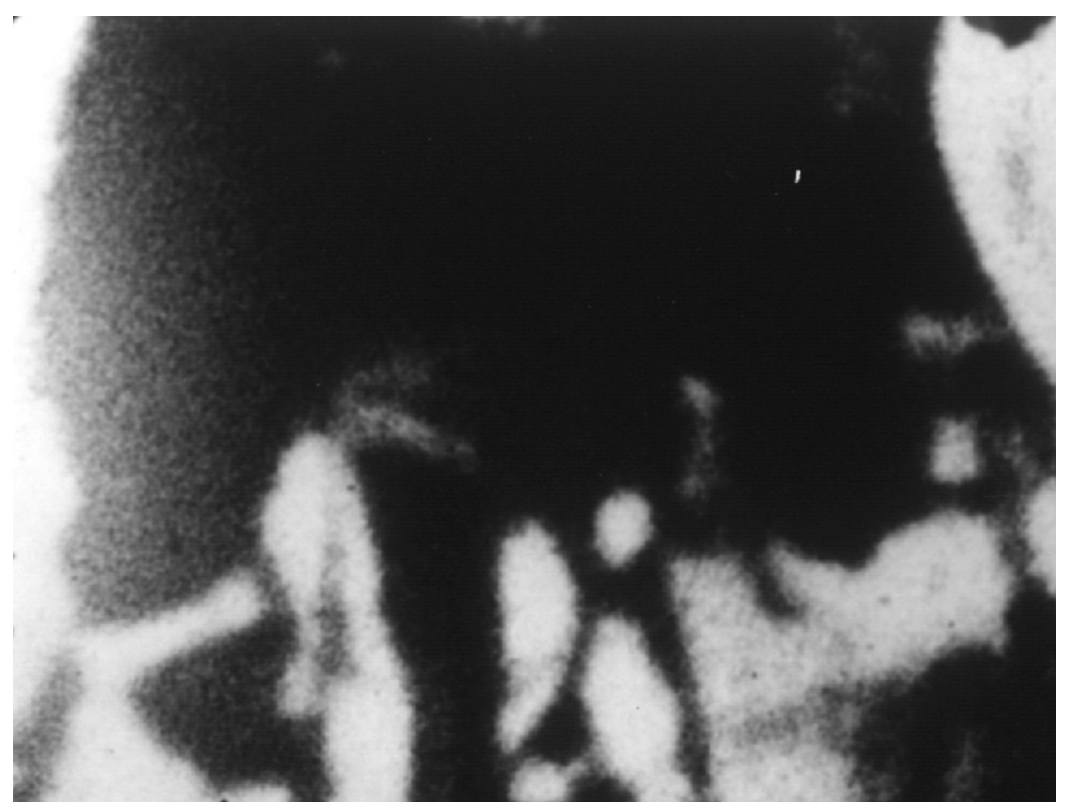

Tom Tom the Piper's Son, Ken Jacobs, 1969-1971

the Piper's Son. Poème élégiaque, Tom Tom procède par fragmentation subtile, tout à la fois de motifs privilégiés mais aussi de détails admirablement arbitraires qui en deviennent des fétiches cinétiques, comme cette robe rayée ou cette échelle posée dans une grange; recherche critique intégrale, au cours de sa trajectoire, il dévoile aussi les failles et les erreurs de tournage (regards à la caméra, décors en carton-pâte, etc.); du ready-made, il retient le principe de monter deux fois tel quel le film d'origine, la seconde reprise étant totalement réinformée par le travail accompli; du cinéma structurel, il reprend le principe selon lequel il faut réaliser un film entièrement réflexif à partir des seules données cinématographiques; il apporte une contribution majeure à la matériologie grâce à l'investigation menée sur les formes du mouvement et notamment sur ce phénomène propre au cinéma aussi irrémédiable que refoulé, la non-fixité de l'image; et il livre l'analyse de film la plus élaborée jamais proposée à ce jour. Tom Tom est un modèle théorique, un laboratoire plastique et une expérience psychique que Ken Jacobs prolongera avec ses performances sur les rapports singuliers que 
l'image cinématographique entretient avec le fonctionnement nerveux, performances effectuées à partir de films Lumière ou d'enregistrements des danses de Loïe Fuller, grande initiatrice du mouvement moderne.

Notons enfin que plusieurs films importants et qui ne manqueraient pas de renouveler la réflexion en matière de recyclage ont à ce jour disparu: Photogénie mécanique de Jean Grémillon (1925), montage sélectif par Grémillon de ses propres documentaires sur les objets industriels dans une perspective de recherche plastique; Le Monde en parade d'Eugen Deslaw (vers 1930), montage sonore d'actualités; ou le Que Viva Mexico de 1950, remontage du film d'Eisenstein accompli par Kenneth Anger à la demande d'Henri Langlois.

Université de Paris I

\section{NOTES}

1. Sur la distinction entre in re et in se, cf. Salvatore Settis, Remplois. Usage et connaissance de l'Antique au Moyen Âge, Paris, Macula (à paraître).

2. Selon l'expression de Martin Barnier dans En route vers le parlant. Histoire économique, technologique et esthétique du cinéma (1926-1934) (Barnier 2002).

3. Reproduit notamment dans Wolman 1981.

\section{RÉFÉRENCES BIBLIOGRAPHIQUES}

Adorno 1979: Theodor W. Adorno, Philosophie de la nouvelle musique, Paris, Gallimard, 1979.

Barnier 2002: Martin Barnier, En route vers le parlant. Histoire économique, technologique et esthétique du cinéma (1926-1934), Liège, Céfal, 2002.

Benoliel 2000 : Bernard Benoliel, "Angela Ricci Lucchi et Yervant Gianikian, les archéologues de la pellicule", Cahiers du Cinéma, avril 2000.

Léger 1976: Fernand Léger, "Notes préparatoires», dans Alain Sayag (dir.), Cinéma dadaïste et surréaliste, Paris, Centre Georges Pompidou, 1976.

Mekas 1972: Jonas Mekas, "On Tom, Tom and Film Translations", dans Movie Journal, New York, Collier Book, 1972.

Wolman 1981 : Gil Wolman, Résumé des chapitres précédents, Paris, Spiess, 1981. 